Received 00th January 20xx, Accepted 00th January $20 x x$

DOI: $10.1039 / x 0 x \times 00000 x$

\title{
Ni-Catalysed Intramolecular [4+4]-Cycloadditions of Bis-dienes towards Eight-membered Fused Bicyclic Systems: A Combined Experimental and Computational Study
}

\author{
Nuria Llorente, ${ }^{\text {} \ddagger}$ Héctor Fernández-Pérez, ${ }^{\text {} \ddagger}$ Antonio Bauzá, ${ }^{c}$ Antonio Frontera*c and Anton Vidal- \\ Ferran*a,b

\begin{abstract}
Detailed investigations on the use of nickel(0)-based catalysts for intramolecular [4+4]-cycloadditions are presented. Nickel(0) complexes derived from electron-rich triarylphosphines proved to be efficient catalysts for intramolecular [4+4]cycloadditions of an array of structurally diverse bis-dienes (10 examples, up to $78 \%$ isolated yield). The reported synthetic methodology leads to cis-eight-membered fused [6.3.0] bicyclic compounds as well as trans- or cis-eight-membered fused [6.4.0] bicyclic systems. Computational studies on the stereo-determining step of the reaction in combination with experimental results demonstrated that the stereochemical outcome is dictated by the length of the chain linking the two diene units and the geometry of the $\mathrm{C}=\mathrm{C}$ double bonds of the substrates.
\end{abstract}

\section{Introduction}

Medium-sized cyclic compounds, and in particular eightmembered fused polycyclic systems, are key structural motifs in a significant number of natural products. ${ }^{1}$ Among the different strategies for the preparation of such compounds, ${ }^{2}$ metalmediated intramolecular [4+4]-cycloadditions are an elegant, efficient, and atom-economical synthetic methodology. Although metal-mediated [4+4]-cycloadditions of two diene units have been known for some time, ${ }^{3}$ it was Wender's research group ${ }^{4}$ who pioneered the use of intramolecular nickel-catalysed [4+4]-cycloadditions for the highly diastereoselective synthesis of complex natural products (or advanced synthetic intermediates thereof). In Wender's studies, the configurations of the substituted carbons in the tether linking the two diene units were responsible for the high diastereoselectivities observed. ${ }^{4}$ The practicality of this approach was demonstrated by preparing $(+)$-asteriscanolide and $( \pm)$-salsolene oxide using this chemistry as the key step. ${ }^{5}$ More recently, Cheung and co-workers have used rhodium(I)

a. Institute of Chemical Research of Catalonia (ICIQ) \& The Barcelona Institute of Science and Technology (BIST), Av. Països Catalans 16, 43007 Tarragona, Spain. Email:avidal@iciq.cat

b.ICREA, Passeig Lluís Companys 23, 08010 Barcelona, Spain.

c. Departament de Química, Universitat de les Illes Balears (UIB), Ctra. de

Valldemossa km 7.5, 07122 Palma de Mallorca, Spain.

+ Electronic Supplementary Information (ESI) available: Experimental procedures, spectral data for known and new compounds, selected crystallographic information and cartesian coordinates of the calculated structures. CCDC 1834976 and 1834977 contains the supplementary crystallographic data for this paper. See DOI: 10.1039/x0xx00000x.

₹ These authors contributed equally to this work. complexes as catalysts in this type of cycloaddition for the efficient preparation of the corresponding cyclooctadiene derivatives, ${ }^{6}$ though harsher reaction conditions than those reported by Wender are required.

Whilst diastereoselective nickel-catalysed intramolecular [4+4]-cycloadditions of bis-dienes have been reported, the study of this nickel chemistry on prochiral bis-dienes containing heteroatoms in the tether between the two diene units remained scarcely explored. ${ }^{4}$ Herein, we wish to report our findings on the development of nickel(0)-based achiral catalysts for intramolecular [4+4]-cycloadditions of structurally diverse prochiral bis-diene substrates (Scheme 1 ). We also aim to disclose the effects of substituents and $\mathrm{C}=\mathrm{C}$ double bond geometries on the outcome of the $[4+4]$-cycloadditions. Computational studies have been performed to identify the relevant transition states of the stereo-determining step and their relative stabilities and consequently provide a rationalisation of the stereochemical outcome of the reaction.

\section{Results and Discussion}

\section{Study of the [4+4]-cycloadditions}

At the onset of our studies, we used oxygen-containing bisdiene 1a as the model substrate, as its preparation was already reported. ${ }^{7}$ The intramolecular [4+4]-cycloaddition of 1 a was 

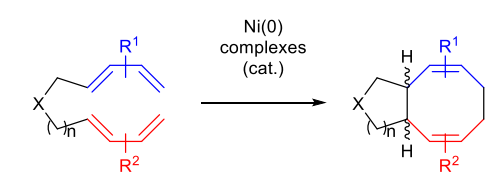

$\mathrm{X}=\mathrm{C}\left(\mathrm{CO}_{2} \mathrm{Me}\right)_{2}, \mathrm{O}, \mathrm{NTs}$

$\mathrm{R}_{1,}, \mathrm{R}_{2}=\mathrm{H}$ or

Scheme 1 Nickel-catalysed intramolecular [4+4]-cycloadditions studied

studied in the presence of catalytic amounts of $\left[\mathrm{Ni}(\operatorname{cod})_{2}\right](\operatorname{cod}=$ 1,5-cyclooctadiene) and a set of phosphorus monodentate ligands (L1-L6; see Table 1 for the structures), in order to test the influence of the ligand on both the reactivity and selectivity of the nickel catalyst. ${ }^{8}$

When $\mathrm{PPh}_{3}$ (L1) was used as the ligand, the cis-fused cyclooctadiene $2 \mathrm{a}$ was obtained in good isolated yields (see entry 1 in Table 1). Under these reaction conditions, one of the possible [4+2]-cycloaddition products ${ }^{9}$ was identified in the crude mixture. We were then interested in studying how the electronic properties of the phosphorus ligands affected the outcome of the cycloaddition reactions. Interestingly, the electron-rich triarylphosphine $\mathbf{L} \mathbf{2}$ provided compound $\mathbf{2 a}$ in almost identical yield to triphenylphosphine and gave only minimal amounts of products 3a (compare entry 2 with entry 1 in Table 1). On the contrary, the conversion of the reaction and selectivity towards product $2 \mathrm{a}$ dropped when the reaction was conducted with ligand $\mathbf{L} \mathbf{3}$, which incorporates sigma electron withdrawing groups (compare entry 3 with entries $1-2$ in Table 1). Likewise, the use of monophosphite ligand $\mathbf{L} 4$ did not significantly improve the previous results and led to decreased activity and yield for cyclooctadiene $\mathbf{2 a}$ (see entry 4 in Table 1 ). As far as the phosphoramidite ligand $\mathbf{L 5}$ is concerned, the corresponding nickel complex proved to be an
Table 1 Screening of ligands for the nickel-catalysed intramolecular [4+4]-cycloaddition of substrate $1 a^{a}$

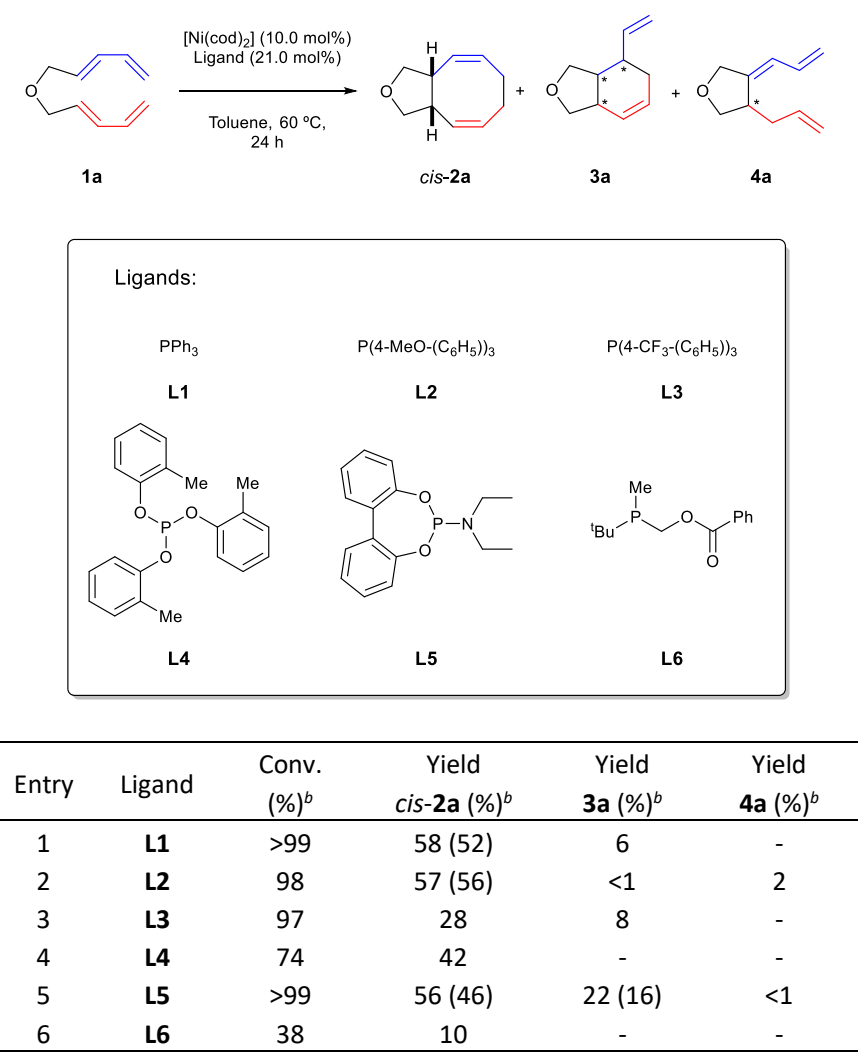

${ }^{a}$ The results are the average of at least two independent runs. ${ }^{b}$ Calculated by ${ }^{1} \mathrm{H}$ NMR spectroscopy of the crude mixture using 1,3,5-trimethoxybenzene as internal standard. In parentheses: isolated yields after column chromatography on silica gel impregnated with $\mathrm{AgNO}_{3}(10 \%)$.

active catalyst for the intramolecular [4+4]-cycloaddition of substrate 1a, with full conversion of 1 a obtained and the product $2 \mathrm{a}$ isolated in a moderate yield $(46 \%$, see entry 5 in Table 1). It is interesting to note that $\mathbf{L 5}$ allowed notable amounts of one of the possible [4+2]-cycloadducts $3 a$ to be isolated and characterised. ${ }^{10}$ Finally, trialkylphosphine ligand $\mathbf{L 6}$ was assessed in this initial screening. As shown in entry 6 (Table $1)$, low reactivity was observed towards the product $2 a$.

The previous results demonstrate that changes in the electronic nature of the ligands lead to significant differences in the yield and selectivity of the cycloaddition reactions. Interestingly, electron-rich triphenylphosphine $\mathbf{L} 2$ was the most selective ligand for the nickel-catalysed intramolecular [4+4]cycloaddition of bis-diene 1a and was selected for further catalytic studies. Thus, with catalytic conditions in hand, we turned our attention to assessing the scope of the nickelcatalysed intramolecular [4+4]-cycloaddition over a set of structurally diverse prochiral bis-diene substrates. First, we focused our attention on bis-dienes $\mathbf{1 b}-\mathbf{1} \mathbf{f},{ }^{7}$ substrates that lead to 10-substituted bicyclo[6.3.0]-undeca-2,6-diene systems (see Table 2 for the structures). The results summarised in Table 2 indicate that the nickel-catalysed cycloadditions to eightmembered fused bicyclic systems were successfully accomplished in almost all cases. Replacement of the oxa group in 1a by a 1,3-dimethoxy-1,3-dioxopropan-2-yl or aza 
substituent was well tolerated and the corresponding [4+4]cycloadducts were isolated in satisfactory yields (see entries 1 and 2 in Table 2). Analysis of the NMR spectra of the eightmembered fused bicyclic systems derived from 1a-1c revealed a cis-fusion between the five- and eight-membered rings. This type of ring fusion was in agreement with previous results in the literature, ${ }^{4 a}$ and was confirmed by $\mathrm{X}$-ray analysis of a single crystal of compound $\mathbf{2 c}{ }^{11}$

We also investigated the outcome of the reaction upon placing a methyl group at the 1-, 2- or 3-position of one of the diene units. Thus, intramolecular [4+4]-cycloadditions of substrates $\mathbf{1 d - 1 f}$ were examined. As shown in Table 2, the selectivities of the cycloadditions were heavily influenced by the substitution pattern of the diene. Introduction of a methyl group at the terminal position of the diene (substrate 1d) was detrimental for the yield: compound $\mathbf{2} \mathbf{d}$ was the only adduct that could be isolated in diastereomerically pure form, but in low isolated yield (13\%, see entry 3 in Table 2$)$. The relative configuration of the three stereogenic carbons in $\mathbf{2 d}$ was established by extensive ${ }^{1} \mathrm{H}$-selective decoupling, 1D NOE and 2D NMR experiments. ${ }^{11}$ Substitution of the diene at the 2position (substrate 1e) was detrimental, as the desired [4+4]cycloadduct could not be detected in the complex mixture of products that was obtained (see entry 4 in Table 2). Finally, substrate $\mathbf{1 f}$ bearing a methyl group at the 3-position cyclised to the cis-fused bicyclic product $\mathbf{2} \mathbf{f}$ in moderate yield (up to $35 \%$ isolated yield, see entry 5 in Table 2). The relative configuration of the two stereogenic carbons in $\mathbf{2} \mathbf{f}$ was established by extensive NMR experiments. ${ }^{11}$
Table 2 Nickel-catalysed intramolecular [4+4]-cycloaddition towards bicyclo[6.3.0] ring systems ${ }^{a}$

Entry

$a$ The results are the average of at least two independent runs. Reactions performed in the presence of $10.0 \mathrm{~mol} \%$ [ $\mathrm{Ni}(\operatorname{cod})_{2}$ ] and $21.0 \mathrm{~mol} \% \mathbf{L 2}$ in toluene at 60 oC for $24 \mathrm{~h} .{ }^{c} 31 \mathrm{~mol} \% \mathrm{~L} 2$ used as ligand. ${ }^{d}$ Not determined. ${ }^{b}$ See note $b$ in Table 1.

Encouraged by these results, we extended the use of this $[4+4]$-cycloaddition strategy to the preparation of 10 substituted bicyclo[6.4.0]dodeca-2,6-diene systems starting from the corresponding bis-dienes $\mathbf{1 g - 1 \mathbf { i }}$ (see Table 3 for the structures). The required substrates were synthesised following well-established synthetic protocols, ${ }^{7}$ though it should be noted that substrate $1 \mathbf{i}$ could only be prepared as a mixture of isomers (E,E-1i:E,Z-1i ratio of 88:12), which were inseparable by standard column chromatography. Under our optimised reaction conditions, substrate $\mathbf{1 g}$ gave [4+4]-cycloadduct $\mathbf{2 g}$ (up to $67 \%$ isolated yield, see entry 1 in Table 3). Spectroscopic data of the cycloaddition product pointed to the trans-eightmembered fused bicyclic system $\mathbf{2 g}$. This type of ring fusion was in agreement with previous results in the literature for comparable compounds, ${ }^{4 c}$ and was unambiguously confirmed by X-ray analysis (Fig. 1).

The same trend was observed for the intramolecular [4+4]cycloaddition of substrates $\mathbf{1 h}$ and $\mathbf{1 i}$, which contained oxa and aza-substituted groups in the chain linking the two diene units. The eight-membered fused bicyclic systems were obtained in good isolated yields ( $61 \%$ and $67 \%$ for $\mathbf{1 h}$ and $\mathbf{1 i}$, respectively; see entries 2 and 3 in Table 3 ). The [4+4]-cycloadducts derived from 1i were obtained as an inseparable mixture of two isomeric compounds in an 88:12 ratio. It is interesting to note the close relationship between the $E, E: E, Z$ isomer ratio of the starting material $\mathbf{1} \mathbf{i}$ and the ratio of the isomeric [4+4]- 
Table 3 Nickel-catalysed intramolecular [4+4]-cycloaddition towards bicyclo[6.4.0] ring systems $^{a}$

Entry

${ }^{a, b}$ See notes $a$ and $b$ in Table 1

cycloadducts (trans-2i and cis-2i), thus suggesting a direct relationship between the geometry of the diene units and the structure of the final products.

In order to fully understand the outcome of the cycloaddition reaction of $\mathbf{1} \mathbf{i}$, the separation of $E, E-\mathbf{1} \mathbf{i}$ and $E, Z-\mathbf{1} \mathbf{i}$ was attempted. The two isomers of $\mathbf{1} \mathbf{i}$ could not be separated by standard column chromatography, but pure samples of $E, E-$ $1 \mathbf{i}$ and $E, Z-1 \mathbf{i}$ could be obtained by semipreparative HPLC. ${ }^{11}$ With $E, E-\mathbf{1} \mathbf{i}$ and $E, Z-\mathbf{1 i}$ in hand, we examined the Ni-catalysed intramolecular [4+4]-cycloadditions employing [ $\left.\mathrm{Ni}(\operatorname{cod})_{2}\right]$ and L2 as catalyst. As shown in Scheme 2, each isomer of substrate 1i exclusively led to one [4+4]-cycloadduct. Whilst $E, E-1 \mathbf{i}$ evolved under the reaction conditions to the trans-eightmembered fused bicyclic system $\mathbf{2} \mathbf{i}$ in high isolated yield (78\%), $E, Z-1 \mathbf{i}$ led to the cis-fused analogue (55\%). Structural assignments were made by analogy with the spectroscopic data of trans-fused product $\mathbf{2 g}$ and by performing extensive NMR experiments on cis-2i. These results illustrate the importance of the geometry of the diene on the outcome of the cycloaddition, as $E, E-\mathbf{1 i}$ and $E, Z-\mathbf{1 i}$ lead to different diastereoisomeric cycloadducts (i.e., trans-2i or cis-2i, respectively).

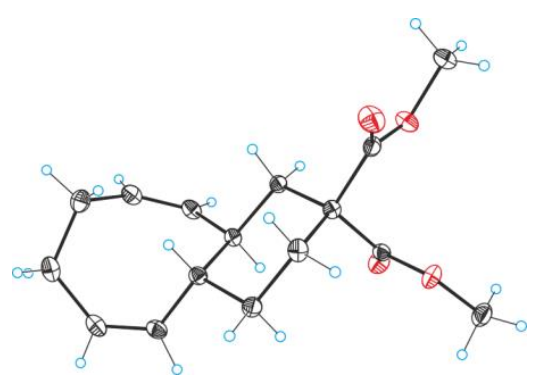

Fig. 1 X-ray crystal structure of trans-2g (ORTEP drawings showing therma ellipsoids at $50 \%$ probability)
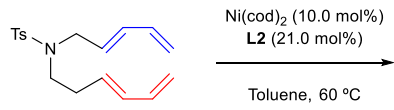

$E, E-1 \mathbf{i}$

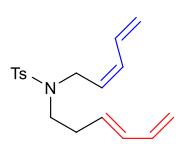

$E, Z-1 \mathbf{i}$

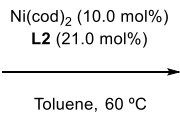

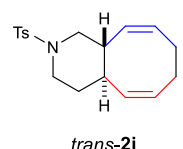

$78 \%$ isolated yield

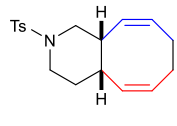

cis-2i

$55 \%$ isolated yield
Scheme 2 Stereospecific nickel-catalysed intramolecular [4+4]-cycloaddition of $\mathbf{1 i}$

\section{Computational studies}

To shed light on the outcome of the [4+4]-cycloaddition reactions, we performed a theoretical investigation (BP-86D3/def2-TZVP, see ESI for details) into the reactivity of substrates $1 \mathbf{a}, E, E-1 \mathbf{h}$ and $E, Z-1 \mathbf{h}$. A tentative reaction pathway for the intramolecular nickel-catalysed [4+4]-cycloadditions of bis-dienes is provided in Scheme 3. The accepted view for this transformation $3 \mathrm{~b}, 3 \mathrm{c}, 3 \mathrm{f}, 4 \mathrm{a}, 4 \mathrm{c}, 12$ is that the nickel precursor reacts with the substrate to form bis-diene nickel complexes $\mathbf{5}$ that, after an oxidative cyclisation of the two internal $\mathrm{C}=\mathrm{C}$ bonds, lead to bis-allyl $\mathrm{Ni}(\mathrm{II})$ complexes $\mathbf{6}$. Reductive elimination from these $\mathrm{Ni}(\mathrm{II})$-complexes 6 leads to the cyclised product and nickel complexes capable of re-entering the catalytic cycle. We have focused our study on the first $\mathrm{C}-\mathrm{C}$ bond formation, where the five- (for substrate 1a) or six-membered ring (for compounds $E, E-\mathbf{1} \mathbf{h}$ and $E, Z-\mathbf{1} \mathbf{h})$ is formed and the stereochemistry of the ring-fusion is defined. We have compared the energies of the transition states (TSs) for the paths leading to the cis- and transcycloadducts for the three substrates incorporated to this computational study.

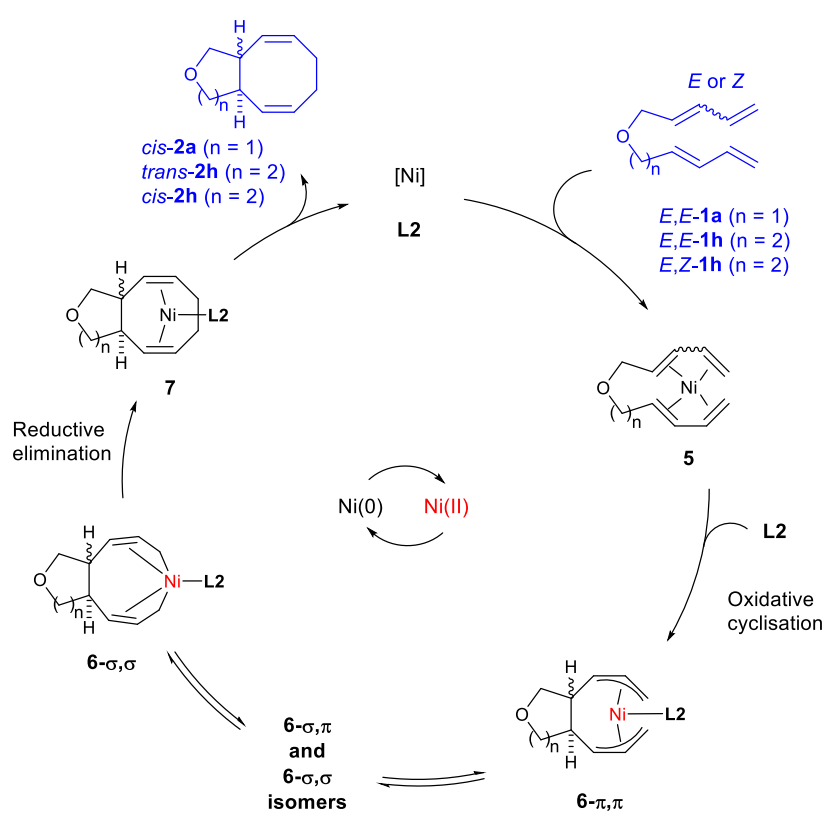


Scheme 3 Tentative reaction pathway rationale for nickel-catalysed intramolecular $[4+4]$-cycloadditions

We first studied the reactivity of compound 1a (see Fig. 2). Experimentally, phosphine $\mathbf{L} \mathbf{2}$ is used in the reaction. However, our computational studies did not incorporate $\mathbf{L 2}$ in the coordination sphere of the $\mathrm{Ni}(0)$ centre as by coordinating to the four double bonds of the substrate it had already accommodated eighteen electrons. We performed a conformational search on the starting complex $[\mathrm{Ni}(\mathbf{1 a})]$ and found two possible binding modes (denoted as pre-TS-1 and pre-TS-2) depending on the relative orientation of the diene units. Remarkably, pre-TS-2 is considerably more stable than pre-TS-1 and it has the adequate orientation to yield the transisomer. Both transition states on the potential hypersurface
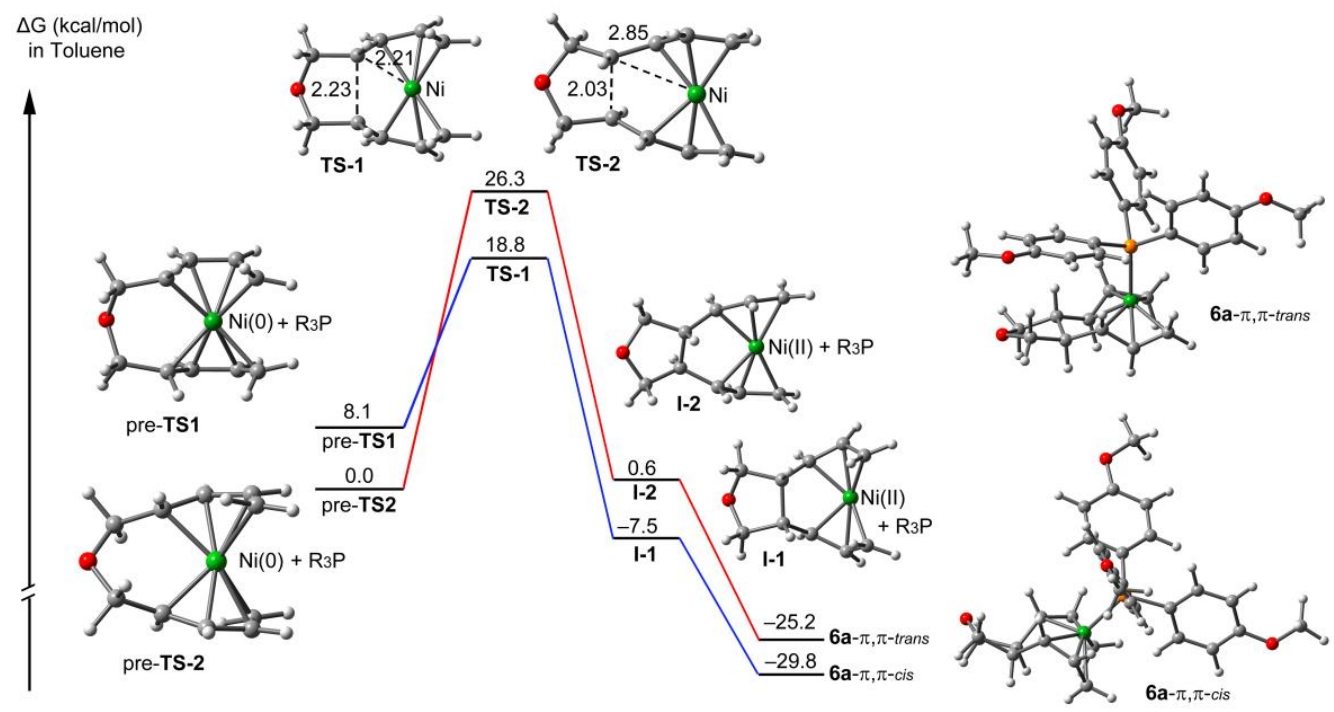

Fig. 2 Optimised geometries BP86-D3/def2-TZVP level of theory of the pre-transition states (pre-TS), transition states (TS), intermediates (I) and products for the reaction of 1a and its energetic profile ( $\mathrm{kcal} / \mathrm{mol})$ in toluene (distances in $\AA$ )

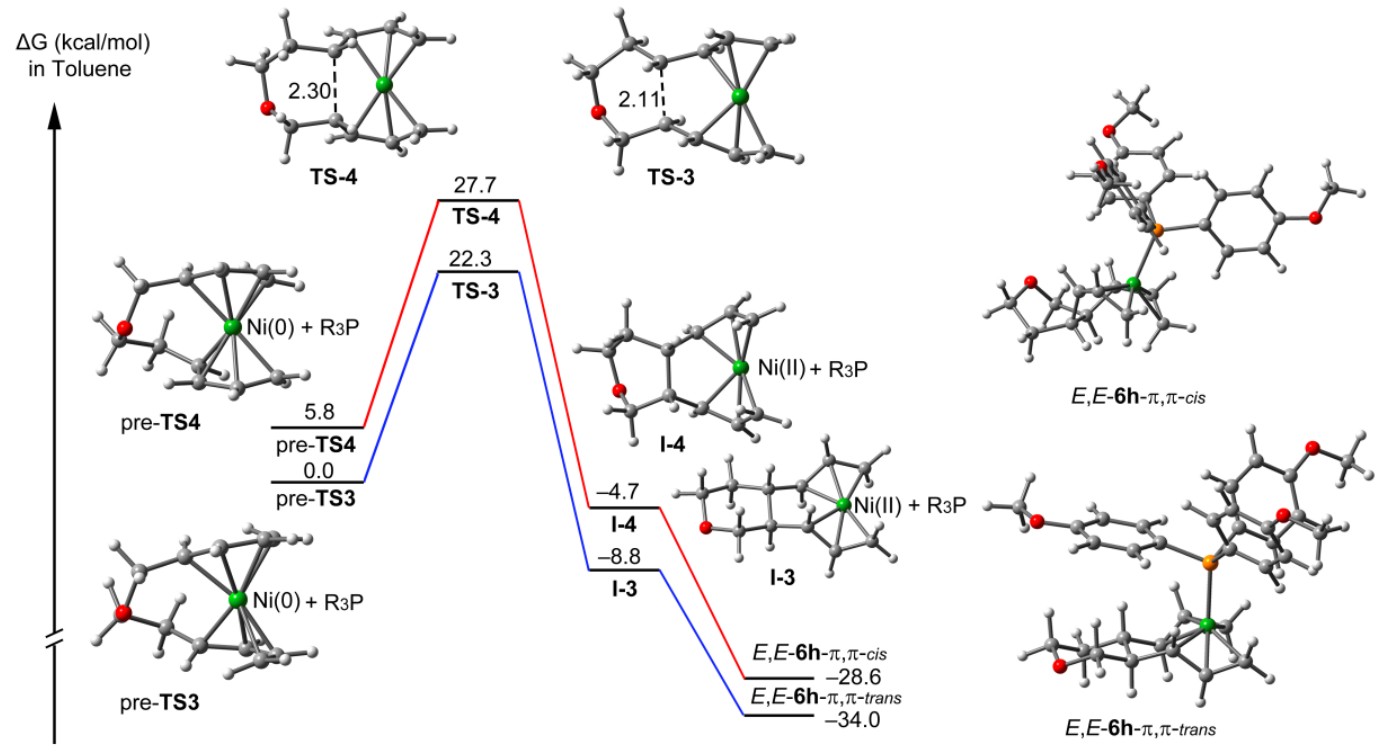

Fig. 3 Optimised geometries BP86-D3/def2-TZVP level of theory of the pre-transition states (pre-TS), transition states (TS), intermediates (I) and products for the reaction of E,E-1h and its energetic profile ( $\mathrm{kcal} / \mathrm{mol}$ ) in toluene (distances in $\AA$ ).

were located and the TS that yields the cis-isomer (denoted as TS-1) is lower in energy than the TS for the trans-isomer (TS-2; see Fig. 2), which is in good agreement with the experimental results. ${ }^{13}$ The reaction barrier is $18.8 \mathrm{kcal} / \mathrm{mol}$ for TS-1 and 26.3 $\mathrm{kcal} / \mathrm{mol}$ for TS-2. The higher stability of TS-1 (i.e. $7.5 \mathrm{kcal} / \mathrm{mol}$ ) can be attributed to the closer proximity of the metal centre to the $\mathrm{C}$ atoms that are forming the $\mathrm{C}-\mathrm{C}$ bond in TS-1 $\left(\mathrm{d}_{\mathrm{N} i \cdots \mathrm{C}}=2.21\right.$ $\AA)$ than in TS-2 $\left(d_{N i \cdots C}=2.85 \AA\right)$. Consequently, the more stable pre-TS-2 trans-isomer needs to isomerize to the less stable preTS-1 cis-isomer prior to the $\mathrm{C}-\mathrm{C}$ bond formation. In the resulting intermediates (denoted as $\mathbf{I - 1}$ and $\mathbf{I - 2}$ in Fig. 2) the nickel(II) centre is able to incorporate the phosphine as an additional 
ligand, stabilising the complexes. The reaction is highly exergonic in both cases.

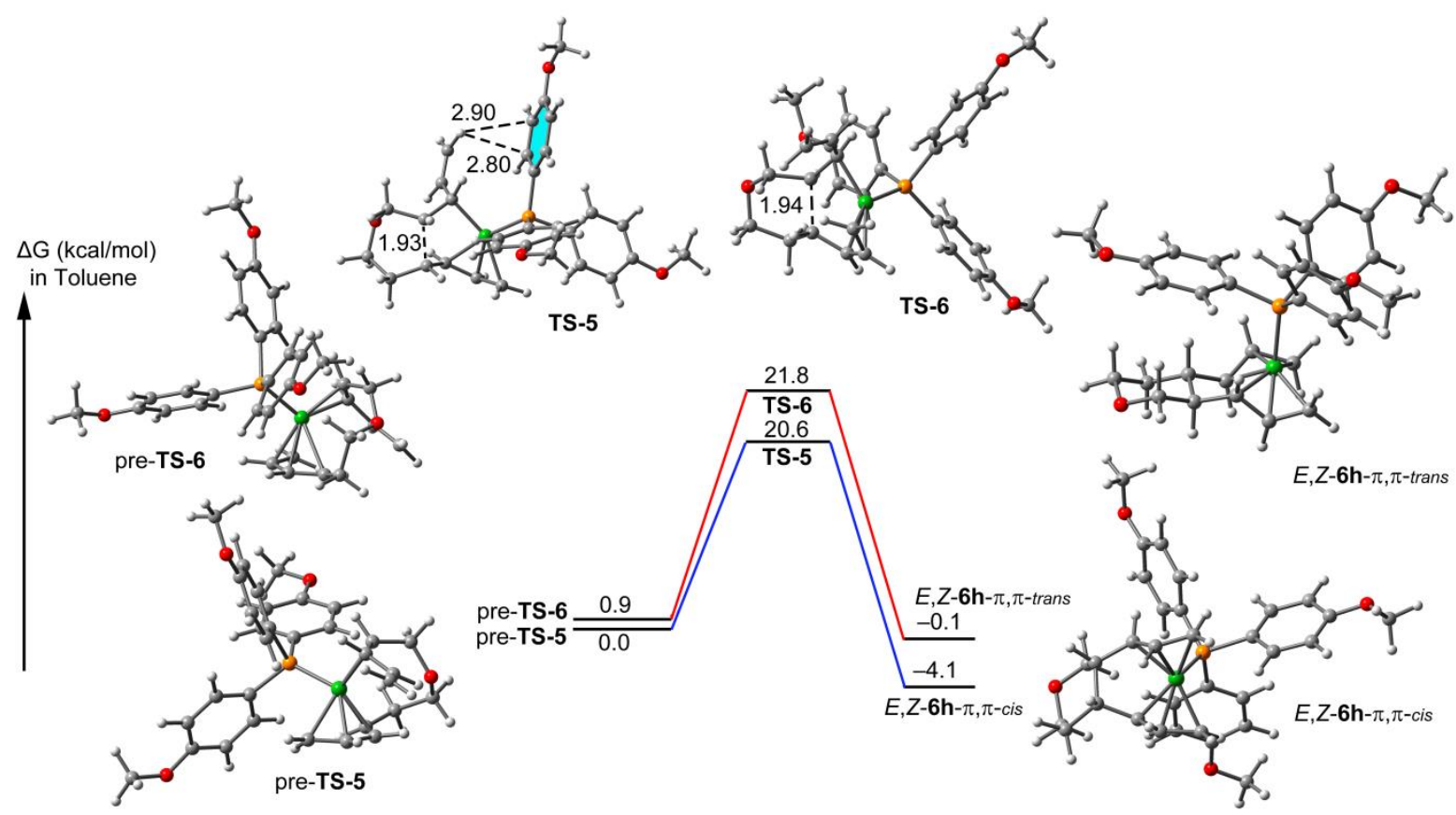

Fig. 4 Optimised geometries BP86-D3/def2-TZVP level of theory of the pre-transition states (pre-TS), transition states (TS), intermediates (I) and products for the reaction of E,Z-1h and its energetic profile $(\mathrm{kcal} / \mathrm{mol})$ in toluene (distances in $\AA$ ).

We have also studied the reactivity of compound $E, E-\mathbf{1 h}$ (see Fig. 3). Experimentally, the cycloaddition of this substrate leads to trans-2h rather than cis-2h (it is interesting to note that the substrate leading to a cis-fusion between the five- and eightmembered rings, i.e. $E, E-1 \mathrm{a}$, and that leading to a trans-fusion between the six- and eight-membered rings, i.e. $E, E-1 \mathbf{h}$, differ in the length of the spacer used to connect both $\pi$-systems). Similarly to compound E,E-1a, we have also performed a conformational study of $[\mathrm{Ni}(E, E-1 \mathbf{h})]$ and the trans-isomer is also the most stable $(5.8 \mathrm{kcal} / \mathrm{mol}$, denoted as pre-TS-3 in Fig. 3). We located both transition states on the potential hypersurface, and, as for the cycloadditions of $\mathbf{1 a}$, we considered that the phosphine does not participate in the process (transition states for the cycloaddition of $E, E-\mathbf{1 h}$ with one uncoordinated terminal $\mathrm{C}=\mathrm{C}$ bond of the substrate and ligand $\mathbf{L} \mathbf{2}$ coordinated to the $\mathrm{Ni}(0)$ centre were located and were higher in energy than those involving coordination of the substrate through the four $\mathrm{C}=\mathrm{C}$ bonds $\left.{ }^{13}\right)$. Gratifyingly, the TS that yields the trans isomer (TS-3) is lower in energy than the TS for the cis isomer (TS-4; see Fig. 3), which is in good agreement with the experimental results. The reaction barrier is 22.3 $\mathrm{kcal} / \mathrm{mol}$ for TS-3 and $27.7 \mathrm{kcal} / \mathrm{mol}$ for TS-4. In this case TS-3 is more stable than TS- $\mathbf{4}$ because the former can adopt a perfect chair conformation in the formation of the six-membered ring (see Figure S3 for a detailed representation of the TSs). In contrast, the TS- 4 adopts a boat-like conformation in the C-C bond formation. The reaction is highly exergonic for both stereoisomers upon coordination of the phosphine.
In order to support the level of theory used herein, we have also computed the energies of the TSs for compounds 1 a and E,E-1h using MP2/def2-TZVP (in toluene) and we have observed the same trend. That is, the TS-1 is lower than the TS-2 for $\mathbf{1 a}$ in $5.6 \mathrm{kcal} / \mathrm{mol}(7.5 \mathrm{kcal} / \mathrm{mol}$ using DFT) and the TS-3 is lower than the TS-4 for $E, E-1 \mathrm{~h}$ in $2.3 \mathrm{kcal} / \mathrm{mol}\left(5.4 \mathrm{kcal} / \mathrm{mol}\right.$ using DFT). ${ }^{14}$

Finally, we studied the most favourable [4+4]-cycloaddition reaction pathway for $E, Z-\mathbf{1 h}$, which has the same spacer as $E, E$ 1h but has one $(Z)$-configured $C=C$ double bond instead of two $(E)$-configured $\mathrm{C}=\mathrm{C}$ bonds. Although the cycloaddition reaction of this substrate was not studied experimentally, ${ }^{15}$ we carried out computational studies with $E, Z-\mathbf{1 h}$ rather than $E, Z-\mathbf{1 i}$ for the sake of consistency with the other bis-dienes studied at the computational level. In this case, the conformational study of [Ni(E,Z-1h)] revealed that both isomers (pre-TS-5 and pre-TS-6) were almost isoenergetic. Moreover, we could not locate any transition state with the four $\mathrm{C}=\mathrm{C}$ bonds coordinated to the nickel centre: the $Z$-configuration of the double bond in $E, Z-1 \mathbf{h}$ hinders the coordination of its terminal double bond to the nickel centre and facilitates the coordination of $\mathbf{L 2}$. Thus, we have located the corresponding transition states with $\mathbf{L} 2$ and three $\mathrm{C}=\mathrm{C}$ bonds coordinated to the nickel centre on the potential hypersurface and, remarkably, the TS that leads to the cis isomer (TS-5) is slightly lower in energy than that for the trans-isomer (TS-6; see Fig. 4). The reaction barrier is 20.6 $\mathrm{kcal} / \mathrm{mol}$ for TS-5 and $21.8 \mathrm{kcal} / \mathrm{mol}$ for TS-6. It should be noted that whilst the computed results are in qualitative agreement with the experimental results, they do not quite match the 
relative magnitudes of the stereoselection (only one stereoisomer is experimentally observed from $E, Z-\mathbf{1} \mathbf{h}$, whilst the energy differences between TS-5 and TS-6 is ca. $1 \mathrm{kcal} / \mathrm{mol}$ ). In spite of TS-6 adopting a perfect chair conformation for the formation of the six-membered ring (see Fig. S4 for a detailed representation of the transition states), TS-5 presents an extra stabilisation energy that comes from the interaction of a $\mathrm{H}$ atom of the uncoordinated double bond with a $\mathrm{C}=\mathrm{C}$ bond of the aromatic ring (see dashed lines in Fig. 4). The non-coordinated double bond in TS-6 is oriented totally different and does not establish any interaction with the phosphine. It should be noted that the reaction is exergonic for the cis isomer $(-4.1 \mathrm{kcal} / \mathrm{mol})$ and thermo-neutral for the trans isomer $(-0.1 \mathrm{kcal} / \mathrm{mol}$ ) (see Fig. 4). These data therefore suggest that the ring fusion is thermodynamically rather than kinetically controlled. Consequently, the formation of $E, Z-6 \mathbf{h}-\pi, \pi$-trans is reversible and the selectivity can be explained by the energy difference between cis and trans isomers $(-4.0 \mathrm{kcal} / \mathrm{mol})$, which correlates with the selectivity observed experimentally.

\section{Conclusions}

In summary, nickel-catalysed intramolecular [4+4]cycloadditions for the preparation of cis-eight-membered fused [6.3.0] bicyclic compounds and trans- or cis- eight-membered fused [6.4.0] bicyclic systems from structurally diverse bisdienes are reported. The catalytic intramolecular [4+4]cycloaddition reaction proceeds efficiently on a set of bis-dienes linked by a three-atom chain to afford cis-configured eightmembered carbocycles fused to a five-membered ring. Analogously, the reported chemistry for bis-dienes linked by a four-atom chain gives access to trans- or cis-configured eightmembered carbocycles fused to a six-membered ring. Computational studies on the stereo-determining step of the reaction have demonstrated that the stereochemical outcome of the reaction is dictated by the length of the chain linking the two diene units, and the geometry of the $\mathrm{C}=\mathrm{C}$ double bonds in the diene units of the substrates. Ongoing research activities are aimed at developing catalytic enantioselective versions of this transformation.

\section{Experimental}

For the general considerations of the experimental and theoretical methods, see the Electronic Supplementary Information (ESI).

\section{General procedure for [4+4]-cycloadditions}

A solution of the substrate $(1.00 \mathrm{mmol})$ and ligand $(0.21 \mathrm{mmol})$ in anhydrous and deoxygenated toluene was prepared under inert atmosphere. [Ni(cod $\left.)_{2}\right](0.10 \mathrm{mmol})$ was added dropwise from a stock solution in anhydrous toluene. In all cases, the molar concentration of a given substrate in the reaction medium was adjusted to $0.20 \mathrm{M}$. The resulting mixture was carefully heated at $60^{\circ} \mathrm{C}$ under an argon atmosphere and stirred for $24 \mathrm{~h}$. The reaction mixture was then allowed to reach room temperature and was exposed to air for $1 \mathrm{~h}$. The mixture was filtered through a short pad of silica and further eluted with diethyl ether. The filtrate was concentrated in vacuo and the resulting crude mixture was analysed by NMR spectroscopy. Purification of the desired product was achieved by column chromatography on silica gel impregnated with silver nitrate (10\%).

\section{Product trans-2i}

Product trans-2i was prepared following the general procedure starting from substrate $E, E-1 \mathbf{i}(0.107 \mathrm{~g}, 0.34 \mathrm{mmol})$, ligand $\mathbf{L 2}$ (25.5 mg, $0.071 \mathrm{mmol})$, and [ $\left.\mathrm{Ni}(\operatorname{cod})_{2}\right](9.5 \mathrm{mg}, 0.034 \mathrm{mmol})$. It was obtained as colourless liquid $\left(0.084 \mathrm{~g}, 78 \%\right.$ yield). ${ }^{1} \mathrm{H}$ NMR $\left(500 \mathrm{MHz}, \mathrm{CDCl}_{3}\right) \delta 7.66-7.64\left(\mathrm{~m}, 2 \mathrm{H}, \mathrm{H}_{\text {arom }}\right), 7.34-7.32(\mathrm{~m}$, $\left.2 \mathrm{H}, \mathrm{H}_{\text {arom }}\right), 5.60-5.55(\mathrm{~m}, 1 \mathrm{H}), 5.53-5.48(\mathrm{~m}, 1 \mathrm{H}), 5.33-5.29$ $(\mathrm{m}, 1 \mathrm{H}), 5.25-5.22(\mathrm{~m}, 1 \mathrm{H}), 3.88-3.82(\mathrm{~m}, 2 \mathrm{H}), 2.72-2.65(\mathrm{~m}$, $1 \mathrm{H}), 2.47-2.37(\mathrm{~m}, 5 \mathrm{H}), 2.27-2.17(\mathrm{~m}, 4 \mathrm{H}), 1.91\left(\mathrm{dd},{ }^{2} \mathrm{~J}_{\mathrm{H}-\mathrm{H}}=3 \mathrm{~J}_{\mathrm{H}-}\right.$ $\mathrm{H}=11.3 \mathrm{~Hz}, 1 \mathrm{H}), 1.85-1.81(\mathrm{~m}, 1 \mathrm{H}), 1.51-1.42(\mathrm{~m}, 1 \mathrm{H}) \mathrm{ppm}$. ${ }^{13} \mathrm{C}\left\{{ }^{1} \mathrm{H}\right\} \mathrm{NMR}\left(125 \mathrm{MHz}, \mathrm{CDCl}_{3}\right) \delta 143.6$ ( $\mathrm{C}_{q}$ arom $), 133.5$ ( $\mathrm{C}_{q}$ arom $)$, $132.4(\mathrm{CH}=), 129.8\left(\mathrm{CH}_{\text {arom }}\right), 129.5(\mathrm{CH}=), 129.4(\mathrm{CH}=), 127.9$ $(\mathrm{CH}=), 127.8\left(\mathrm{CH}_{\text {arom }}\right), 51.7\left(\mathrm{CH}_{2}\right), 46.7\left(\mathrm{CH}_{2}\right), 42.3(\mathrm{CH}), 41.8$ (CH), $32.3\left(\mathrm{CH}_{2}\right), 27.9\left(\mathrm{CH}_{2}\right), 27.8\left(\mathrm{CH}_{2}\right), 21.7\left(\mathrm{CH}_{3}\right)$ ppm. HRMS $\left(\mathrm{ESI}^{+}\right): \mathrm{m} / \mathrm{z}$ calcd for $\mathrm{C}_{18} \mathrm{H}_{24} \mathrm{NO}_{2} \mathrm{~S}[\mathrm{M}+\mathrm{H}]^{+} 318.1522$, found 318.1519 .

\section{Product cis-2i}

Product cis-2i was prepared following the general procedure starting from substrate $E, Z-1 \mathbf{i}(15.0 \mathrm{mg}, 0.0473 \mathrm{mmol})$, ligand $\mathbf{L} \mathbf{2}$ (3.57 mg, $0.0099 \mathrm{mmol})$, and [Ni(cod) $)_{2}$ ( $\left.1.33 \mathrm{mg}, 0.0047 \mathrm{mmol}\right)$. It was obtained as colourless liquid ( $8.3 \mathrm{mg}, 55 \%$ yield). ${ }^{1} \mathrm{H}$ NMR $\left(400 \mathrm{MHz}, \mathrm{CDCl}_{3}\right) \delta 7.65-7.62(\mathrm{~m}, 2 \mathrm{H}), 7.33-7.31(\mathrm{~m}, 2 \mathrm{H}), 5.61$ $-5.51(\mathrm{~m}, 3 \mathrm{H}), 5.20-5.16(\mathrm{~m}, 1 \mathrm{H}), 3.52-3.47(\mathrm{~m}, 1 \mathrm{H}), 3.31-$ $3.25(\mathrm{~m}, 2 \mathrm{H}), 2.74-2.52(\mathrm{~m}, 5 \mathrm{H}), 2.43(\mathrm{~s}, 3 \mathrm{H}), 2.14-1.97(\mathrm{~m}$, 2H), $1.67-1.58(\mathrm{~m}, 2 \mathrm{H}) \mathrm{ppm} .{ }^{13} \mathrm{C}\left\{{ }^{1} \mathrm{H}\right\} \mathrm{NMR}\left(100 \mathrm{MHz}, \mathrm{CDCl}_{3}\right) \delta$ 143.5 ( $\left.C_{q \text { arom }}\right), 133.4$ ( $\left.\mathrm{C}_{\text {q arom }}\right), 132.1(\mathrm{CH}=), 129.8\left(\mathrm{CH}_{\text {arom }}\right), 128.9$ $(\mathrm{CH}=), 128.3(\mathrm{CH}=), 128.2(\mathrm{CH}=), 127.8\left(\mathrm{CH}_{\text {arom }}\right), 49.7\left(\mathrm{CH}_{2}\right), 45.6$ $\left(\mathrm{CH}_{2}\right), 39.6(\mathrm{CH}), 37.8(\mathrm{CH}), 29.1\left(\mathrm{CH}_{2}\right), 29.0\left(\mathrm{CH}_{2}\right), 26.9\left(\mathrm{CH}_{2}\right)$, $21.7\left(\mathrm{CH}_{3}\right) \mathrm{ppm}$. All spectroscopic data were in agreement with those previously reported in the literature. ${ }^{6}$

\section{Conflicts of interest}

There are no conflicts to declare.

\section{Acknowledgements}

We thank MINECO (CTQ2014-60256-P, CTQ2017-89814-P, CTQ2014-57393-C2-1-P and CTQ2017-85821-R FEDER funds and Severo Ochoa Excellence Accreditation SEV-2013-0319) and the ICIQ Foundation for financial support. N. Llorente thanks the ICIQ Foundation for a predoctoral fellowship. We are grateful to J. Benet-Buchholz for X-ray crystallographic data and Ms. R. Somerville for proof reading the manuscript.

\section{Notes and references}


1 See, for example: (a) N. Blanchard and J. Eustache, in Metathesis in Natural Product Synthesis: Strategies, Substrates and Catalysts, eds. J. Cossy, S. Arseniyadis and C. Meyer, Wiley-VCH Verlag GmbH \& Co. KGaA, 2010, ch. 1, pp. 1-43; (b) Y. Wang and Z.-X. Yu, Acc. Chem. Res., 2015, 48, 2288.

2 For general reviews, see: (a) G. Kaupp, Angew. Chem., Int. Ed. Engl., 1992, 31, 422; (b) S. M. Sieburth and N. T. Cunard, Tetrahedron, 1996, 52, 6251; (c) Z.-X. Yu, Y. Wang and Y. Wang, Chem. - Asian J., 2010, 5, 1072.

3 (a) H. W. B. Reed, J. Chem. Soc. 1954, 1931; (b) W. Brenner, P. Heimbach, H. Hey, E. W. Müller and G. Wilke, Liebigs Ann. Chem., 1969, 727, 161; (c) P. W. N. M. Van Leeuwen and C. F. Roobeek, Tetrahedron, 1981, 37, 1973; (d) H. tom Dieck and J. Dietrich, Chem. Ber., 1984, 117, 694; (e) H. tom Dieck, J. Dietrich and G. Wilke, Angew. Chem., Int. Ed. Engl., 1985, 24, 781; (f) A. Tenaglia, P. Brun and B. Wägell, J. Organomet. Chem., 1985, 285, 343; (g) M. Mallien, E. T. K. Haupt and H. tom Dieck, Angew. Chem., Int. Ed. Engl. 1988 27, 1062; (h) K. U. Baldenius, H. Tom Dieck, W. A. König, D. Icheln and T. Runge, Angew. Chem., Int. Ed. Engl., 1992, 31, 305.

4 (a) P. A. Wender and N. C. Ihle, J. Am. Chem. Soc., 1986, 108, 4678; (b) P. A. Wender and M. L. Snapper, Tetrahedron Lett., 1987, 28, 2221; (c) P. A. Wender and N. C. Ihle, Tetrahedron Lett., 1987, 28, 2451; (d) P. A. Wender and M. J. Tebbe, Synthesis, 1991, 1089; (e) P. A. Wender, J. Nuss, D. B. Smith, A. Suarez-Sobrino, J. Vågberg, D. Decosta and J. Bordner, J. Org. Chem., 1997, 62, 4908.

5 (a) P. A. Wender, N. C. Ihle and C. R. D. Correia, J. Am. Chem. Soc., 1988, 110, 5904; (b) P. A. Wender, M. P. Croatt and B. Witulski, Tetrahedron, 2006, 62, 7505.

6 J. W. Park, J. E. Park, J. H. Park, M. R. Hong, S. M. Kim, Y. K. Chung and C. H. Kim, Synlett, 2016, 27, 455.

7 Its reported preparation method involved using (E)-5chloropenta-1,3-diene with small amounts of the cis-isomer (ratio $E, E: E, Z$ isomers $>95: 5)$. For the preparation of substrate 1a, see also: (a) R. Hertel, J. Mattay and J. Runsink, J. Am. Chem. Soc., 1991, 113, 657; for the preparation of substrate $\mathbf{1 b}$, see also: (b) J. M. Takacs and E. C. Lawson, Organometallics 1994, 13, 4787; for the preparation of substrate 1c, see also: (c) M. Takimoto and M. Mori, J. Am. Chem. Soc. 2002, 124, 10008.

8 The reaction of substrate $\mathbf{1 a}$ exclusively in the presence of $\mathrm{Ni}(\mathrm{cod})_{2}(10.0 \mathrm{~mol} \%)$ led to a complex mixture of unidentified products at $90 \%$ conversion.
9 Given the small amounts of product, no attempts were made to elucidate the relative stereochemistry of the stereogenic carbons. 10 In particular, the [4+2] cycloaddition product $\mathbf{3 a}$ using the phosphoramidite ligand $\mathbf{L 5}$ corresponds to $(3 \mathrm{a} R, 4 R, 7 \mathrm{a} S)$-4-vinyl1,3,3a,4,5,7a-hexahydroisobenzofuran (see Figures S33 and S34 for details), whose spectroscopic data are in good agreement with those previously reported in the literature (see reference $7 a$ ).<smiles>C=CC1CC=CC2COCC12</smiles>

11 See the Electronic Supplementary Information (ESI) for details.

12 K. Masanari and Y. Tamaru, in Modern Organonickel Chemistry, ed. Y. Tamaru, Wiley-VCH Verlag GmbH \& Co. KGaA: Weinheim, 2005, ch. 5, pp 137-170.

13 Transition states for the cycloaddition reaction of $E, E-1 \mathrm{~h}$ with with one uncoordinated terminal $\mathrm{C}=\mathrm{C}$ bond of the substrate and ligand $\mathbf{L} 2$ coordinated to the $\mathrm{Ni}(0)$ centre were located (see the structures below and Figure S5 for details). The energies of the new TSs (TS-5 ${ }^{\prime}{ }_{\text {cis }}$ and TS- $\mathbf{6}^{\prime}{ }_{\text {trans, }}$ which lead to cis-2h and trans- $\mathbf{2 h}$, respectively) were higher in energy (i.e., TS-5 ${ }^{\prime}{ }_{\text {is }}=27.3 \mathrm{kcal} / \mathrm{mol}$ and TS- $\left.6_{\text {trans }}=27.9 \mathrm{kcal} / \mathrm{mol}\right)$ than those involving coordination of the substrate through the four $\mathrm{C}=\mathrm{C}$ bonds) (i.e., $\mathrm{TS}-5_{\text {cis }}=22.3 \mathrm{kcal} / \mathrm{mol}$ and TS-6 $6_{\text {trans }}=27.7 \mathrm{kcal} / \mathrm{mol}$ ).
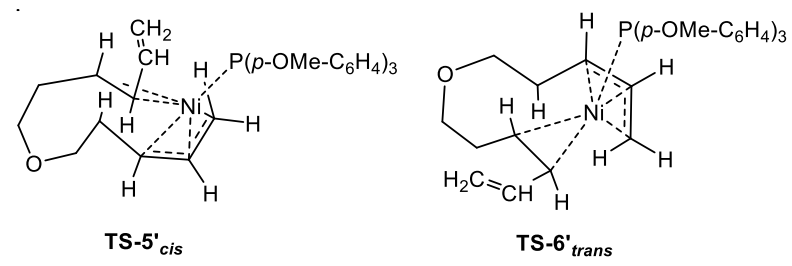

14 The calculations at the MP2 level have not been performed in $E, Z-1 \mathbf{i}$ due to the size of the system incorporating $\mathbf{L} \mathbf{2}$ in the calculations.

15 Substrate $E, Z-1 \mathbf{h}$ is contained as a minor impurity in the samples of substrate $E, E-1 \mathbf{h}(<5 \%$, see reference 7$)$. Accordingly, the [4+4]cycloadduct derived from $E, Z-\mathbf{1}$ (i.e. cis-2h) was present in the corresponding amounts in the cycloaddition reaction mixture and could be separated in analytically pure form by semipreparative HPLC (see the ESI for details). 